DOI: $10.1590 / 1809-6891 v 15 i 220309$

\title{
DESCRIÇÃO DO PLEXO BRAQUIAL DO CACHORRO-DO-MATO (Cerdocyon thous LINNAEUS, 1766)
}

\author{
LUANE LOPES PINHEIRO ${ }^{1}$, ÉRIKA BRANCO ${ }^{2}$, DAMAZIO CAMPOS SOUZA ${ }^{3}$, LUIZA HELENA CORRÊA \\ PEREIRA $^{4}$, ANA RITA LIMA ${ }^{2}$

\begin{abstract}
${ }^{1}$ Pós-Graduanda da Universidade Federal Rural da Amazônia, Belém, PA, Brasil - ana.lima@ufra.edu.br
${ }^{2}$ Professoras Doutoras da Universidade Federal Rural da Amazônia, Belém, PA, Brasil.

${ }^{3}$ Pós-Graduando da Universidade Estadual Paulista Julio de Mesquita Filho, Jaboticabal, SP. Brasil.

${ }^{4}$ Médica Veterinária autônoma, Belém, PA, Brasil
\end{abstract}

\begin{abstract}
O Cerdocyon thous é um canídeo que possui ampla distribuição na América do Sul e, salvo aspectos gerais, têm sua morfologia pouco conhecida na literatura, principalmente no que tange ao sistema nervoso. Com o objetivo de elucidar a composição anatômica do plexo braquial, estudaram-se três exemplares machos provenientes de Paragominas-PA, doados após morte por atropelamento ao Laboratório de Pesquisa Morfológica Animal (LaPMA), da Universidade Federal Rural da Amazônia (UFRA). Os animais foram fixados em solução aquosa de formol 10\% para posterior realização da

dissecação bilateral da origem do plexo braquial. O plexo braquial do $C$. thous é derivado dos três últimos nervos espinhais cervicais e do primeiro nervo torácico (C6-T1). Os principais nervos que o compõem, com suas respectivas origens foram: n. supraescapular, n. subscapular e $n$. musculocutâneo (C6-C7), n. axilar (C7-C8), n. radial (C7T1 e C7-C8), n. mediano, n. ulnar, n. toracodorsal e n. torácico lateral (C8-T1). Concluímos que o plexo braquial do $C$. thous assemelha-se ao descrito para os cães domésticos, apresentando pequenas diferenças quanto à composição de alguns nervos.
\end{abstract}

PALAVRAS-CHAVE: anatomia; Cerdocyon thous; plexo braquial.

\section{DESCRIPTION OF PLEXUS BRACHIAL OF CRAB-EATING FOXES (Cerdocyon thous LINNAEUS, 1766)}

\section{ABSTRACT}

The Cerdocyon thous is a canid that has a wide distribution in South America and, besides some general aspects, its morphology is little known in the literature, especially regarding the nervous system. With the aim of elucidating the anatomical composition of brachial plexus, we studied three male specimens from Paragominas-PA, donated to the Morphological Laboratory of Animal Research (LaPMA), Federal Rural University of Amazonia (UFRA), after death by trampling. The animals were fixed in an aqueous solution of $10 \%$ formaldehyde for bilateral dissection of the origin of the brachial plexus. The brachial

KEYWORDS: anatomy; brachial plexus; Cerdocyon thous. plexus of $C$. thous is derived from the last three cervical nerves and the first thoracic nerve (C6-T1). The main nerves that compose it, with their respective origins were the suprascapular nerve, subscapular nerve and musculocutaneous nerve (C6-C7), axillary nerve (C7-C8), radial nerve (C7-T1 and $\mathrm{C} 7-\mathrm{C} 8)$, median nerve, ulnar nerve, thoracodorsal and thoracic lateral nerve (C8-T1). We conclude that the brachial plexus of $C$. thous is similar to that described for the domestic dogs, showing small differences in the composition of some nerves. 


\section{INTRODUÇÃO}

O cachorro-do-mato (Cerdocyon thous Linnaeus, 1766) é um dos membros da família Canídae mais amplamente distribuído pela América do Sul, podendo ser encontrado no norte da Colômbia e Argentina, na Bolívia nas encostas a leste dos Andes, em todo Paraguai, Uruguai e Venezuela, nas Guianas e Suriname com poucos registros e no Brasil com exceção de parte da Amazônia ${ }^{1}$.

É um animal oportunista de hábitos noturnos e crepusculares, vive solitário ou em pares, explorando áreas de mata e/ou savana ${ }^{2}$. Sua dieta varia sazonalmente: na estação chuvosa frutos e artrópodes são os itens mais frequentes em sua alimentação, ao passo que na estação de seca os pequenos mamíferos são mais frequentes ${ }^{3}$.

As suas principais características são a pelagem curta de coloração cinza, com tonalidades amareladas e uma linha preta na região dorsal que se estende da nuca até a cauda, as extremidades dos membros e as pontas das orelhas também adotam essa coloração ${ }^{2}$. É um canídeo de médio porte que pesa aproximadamente 4 a $7 \mathrm{~kg}$, seu período de gestação é de 56 dias e seus filhotes recebem cuidados de ambos os pais ${ }^{4}$.

De maneira geral, o plexo braquial nas espécies domésticas é descrito como uma rede de nervos que por sua vez são compostos por fibras nervosas sensitivas e motoras, que inervam os membros torácicos e a musculatura das paredes laterais do tórax. Este plexo resulta de ligações estabelecidas entre os ramos ventrais dos últimos três nervos espinhais cervicais e dos dois primeiros torácicos, às vezes com contribuição do quinto nervo cervical ${ }^{5,6}$.

$\mathrm{Na}$ região cervical, o primeiro nervo projeta-se por meio do forame vertebral do atlas e os seis seguintes surgem antes da vértebra de mesma contagem. Assim, o último nervo cervical surge entre a última vértebra cervical e a primeira torácica, de tal forma que o oitavo nervo cervical (C8) é dado na sétima vértebra cervical ${ }^{7}$.

Variações nos níveis de origem do plexo braquial podem estar relacionadas com fatores embriológicos, representados pela posição na qual os brotos dos membros se desenvolvem em relação ao neuro-eixo ${ }^{8}$; assim, quanto mais cefálica for à relação, mais craniais serão os ramos que participaram da formação do plexo.

As espécies que canídeos silvestres, salvo aspectos gerais, têm sua morfologia pouco conhecida na literatura, principalmente quando buscamos dados referentes ao sistema nervoso. Frente à escassez de dados sobre anatomia desta espécie, e com objetivo de contribuir para o conhecimento da neuroanatomia de animais silvestres, o presente trabalho pretendeu elucidar a composição anatômica do plexo braquial do cachorro-do-mato (Cerdocyon thous).

\section{MATERIAL E MÉTODOS}

Foram estudados três exemplares machos de cachorro-do-mato (Cerdocyon thous), que foram a óbito por atropelamento, provenientes de Minas de Bauxita Paragominas-PA, Brasil/Empresa Terra LTDA, sob autorização SEMA-PA Nº $140, \mathrm{~N}^{\circ} 108$ e $\mathrm{N}^{\circ} 522$ doados ao Laboratório de Pesquisa Morfológica Animal, da Universidade Federal Rural da Amazônia (UFRA).

Os animais foram fixados em solução aquosa de formol a $10 \%$ e conservados nesta mesma solução por um período de sete dias. Transcorrido esse prazo, iniciou-se a dissecação bilateral, segundo os planos habituais de incisão que partiram da pele, até atingir o plexo braquial no espaço axilar. As incisões foram feitas próximas ao esterno, seguidas de afastamento dos membros torácicos, ampliando a região axilar para facilitar o acesso.

Para melhor visualização dos ramos ventrais cervicais, foi necessária a dissecação da musculatura próxima aos forames intervertebrais. Além disso, realizou-se a desarticulação das costelas com retirada do esterno e completa evisceração da cavidade torácica, possibilitando a observação dos nervos torácicos, bem como a identificação dos ramos ventrais dos nervos espinhais cervicais e torácicos que participaram da formação do plexo braquial.

Toda nomenclatura adotada foi baseada na Nomenclatura Anatômica Veterinária9 .

\section{RESULTADOS}

Quanto à origem do plexo braquial do cachorro-do-mato, ficou evidenciado um padrão de formação constituído pelos ramos ventrais do sexto (C6), sétimo (C7) e oitavo (C8) nervos espinhais cervicais e primeiro torácico (T1) (Figura 1). Como principais constituintes do plexo braquial, encontramos os nervos: supra-escapular, subescapular, axilar, radial, musculocutâneo, mediano, ulnar, toracodorsal, torácico lateral.

Cienc. anim. bras., Goiânia, v.15, n.2, p. 213-219, abr./jun. 2014 


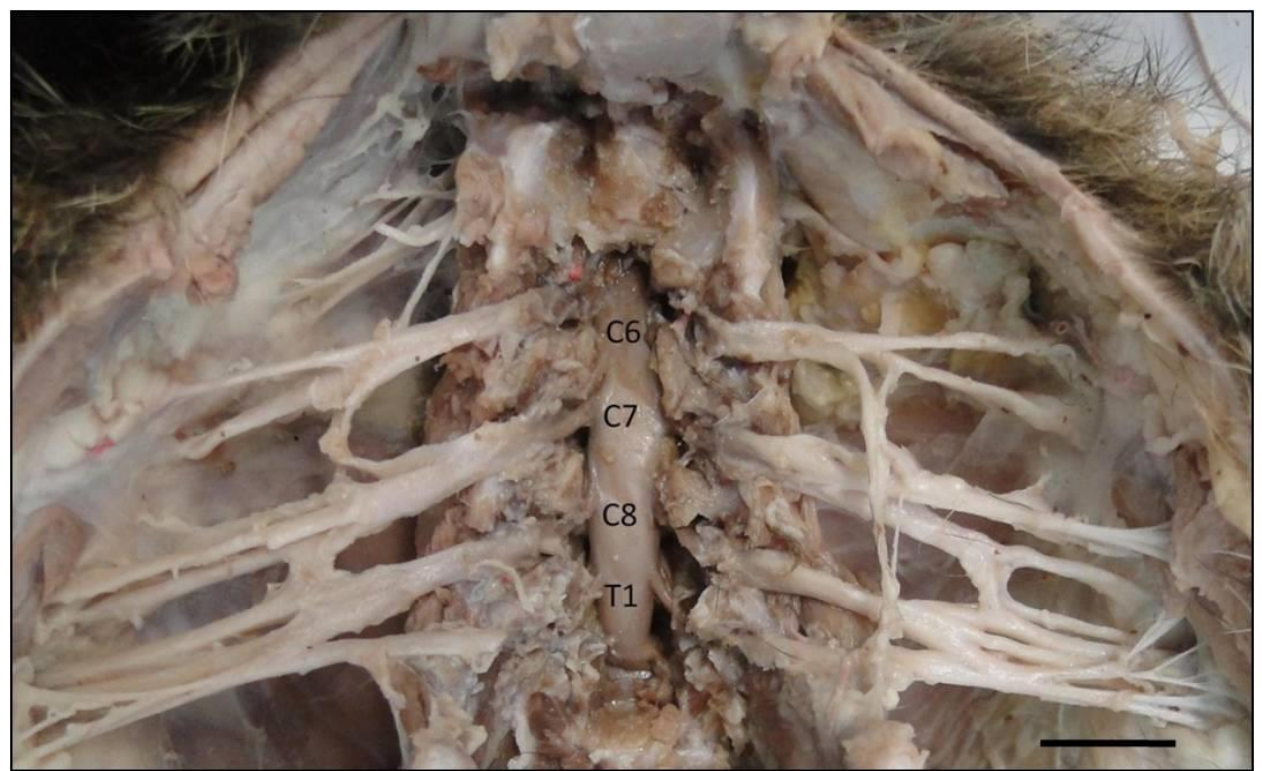

Figura 1: Fotomacrografia da vista ventral do plexo braquial do Cerdocyon thous. Observar: raízes nervosas do sexto, sétimo e oitavo nervos cervicais (C6, C7 e C8) e primeiro nervo torácico (T1). Barra de escala: 1cm.

Nos três exemplares, o nervo supra-escapular origina-se do sexto e sétimo nervos espinhais cervicais (C6 e C7), assim como o nervo subescapular e o nervo musculocutâneo. Já o nervo axilar surge como ramo do sétimo e do oitavo nervos espinhais cervicais (C7 e C8) combinados. O nervo radial procede dos dois últimos nervos espinhais cervicais e do primeiro torácico $(\mathrm{C} 7, \mathrm{C} 8$ e $\mathrm{T} 1)$ em dois exemplares, enquanto que no terceiro não observamos contribuição de T1. Os nervos mediano e ulnar originam-se de um tronco comum do oitavo nervo cervical e do primeiro torácico (C8 e T1). Por fim, o nervo toracodorsal surge a partir de $\mathrm{C} 8$ e $\mathrm{T} 1$ da mesma forma que o nervo torácico lateral (Figura 2 e 3 ).

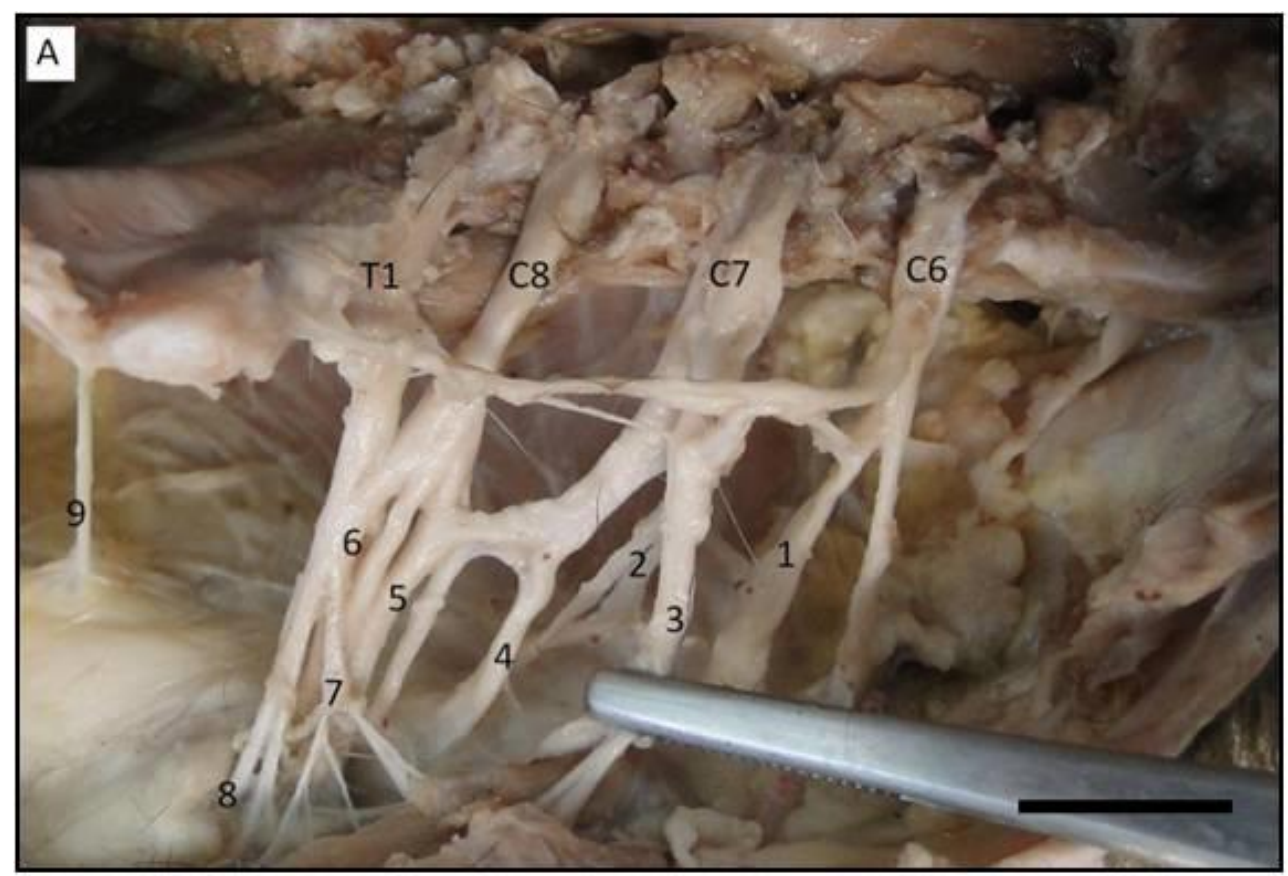

Figura 2: Fotomacrografia da vista ventral do plexo braquial do Cerdocyon thous. Observar: nervo supraescapular (1), nervo subescapular (2), nervo musculocutâneo (3), nervo axilar (4), nervo radial (5), tronco comum para os nervos mediano e ulnar (6), nervo toracodorsal (7), nervo torácico lateral (8) e ramos musculares (9). Barra de escala em A e B: $1 \mathrm{~cm}$. 


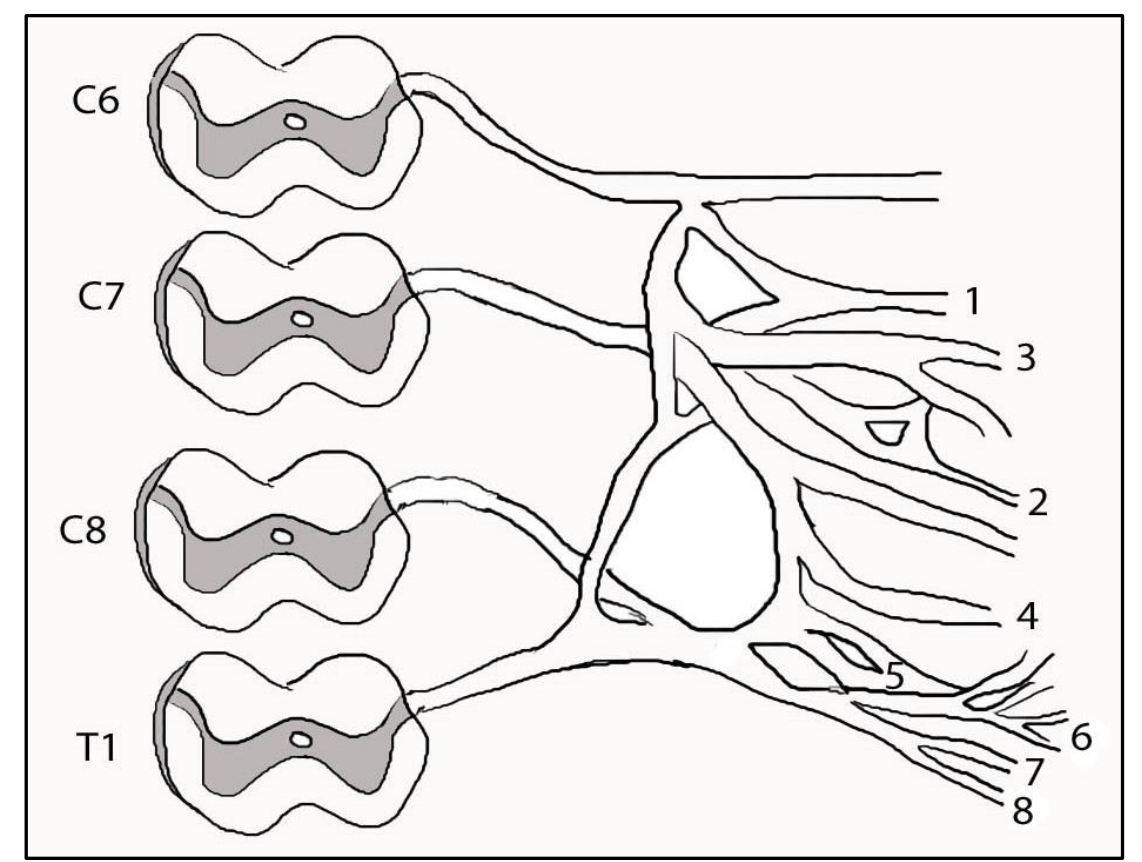

Figura 3: Desenho esquemático do plexo braquial do Cerdocyon thous. Observar o nervo supraescapular (1), nervo subescapular (2), nervo musculocutâneo (3), nervo axilar (4), nervo radial (5), nervo toracodorsal (6), nervo mediano (7) e ulnar (8).

\section{DISCUSSÃO}

O plexo braquial do cachorro-do-mato, constituído pelos três últimos nervos espinhais cervicais $(\mathrm{C} 6, \mathrm{C} 7$ e C8) e pelo primeiro torácico (T1), revelou um padrão semelhante ao descrito para os mamíferos domésticos; entretanto, nestes observa-se a participação também do segundo nervo espinhal torácico $(\mathrm{T} 2)^{5-7}$. Algumas vezes, o quinto nervo cervical participa da formação do plexo braquial e a contribuição do segundo nervo torácico fica, então, reduzida ou ausente ${ }^{6}$.

A origem do plexo braquial do cachorro-domato (Cerdocyon thous), em que o plexo se inicia a partir do sexto nervo espinhal cervical (C6), assemelha-se à dos cães domésticos ${ }^{10} \mathrm{e}$ à do cachorrodo-mato-de-orelhas-curtas (Atelocynus microtis) ${ }^{11}$; entretanto, Allam et al. ${ }^{12}$, realizando dissecações em 58 cães, observou a participação do ramo ventral de C5 em 24 animais, $09 \%$ dos casos. No que diz respeito aos mamíferos silvestres, plexo braquial do cachorrodo-mato (Cerdocyon thous) assemelha-se ao dos catetos $^{13}$, chinchilas ${ }^{14}$ e mocós ${ }^{15}$ e se diferencia do tamanduá-mirim ${ }^{16}$, paca $^{17}$, macaco-prego ${ }^{18}$, porco-

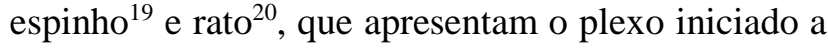
partir do quinto cervical (C5), e dos monotremados (equidna e ornitorrinco) $^{21}$, gorilas, chimpanzés, gibão $^{22}$ e capivara ${ }^{23}$, os quais apresentam origem do plexo em $\mathrm{C} 4$, além da preguiça-de-bentinho ( $B$. tridactylus $)^{24}$ e preguiça-de-coleira (B. torquatus $)^{25}$, que apresentam a origem do plexo braquial a partir de C7.

Quanto ao segmento final que dá origem a este plexo, a espécie estudada apresenta semelhança ao que foi encontrado em cachorro-do-mato-deorelhas-curtas ${ }^{11}$, tamanduá-mirim ${ }^{16}$, macaco-prego ${ }^{18}$, chinchilas ${ }^{14}$ e rato ${ }^{20}$, cujo segmento final corresponde à primeira vértebra torácica (T1). Diferentemente ao descrito para cães domésticos ${ }^{10}$, preguiça-decoleira $^{25}$, pacas $^{17}$, catetos $^{13}$, porco-espinho ${ }^{19}$, mocós ${ }^{15}$ e monotremados ${ }^{21}$, em que esse segmento correspondente à segunda vértebra torácica (T2).

O nervo supra-escapular do Cerdocyon thous apresentou a mesma origem que a descrita para cães ${ }^{10}$, cachorro-do-mato-de-orelhas-curtas ${ }^{11}$ e catetos ${ }^{13}$, nos quais tal nervo se origina das raízes ventrais cervicais C6 e C7. Os autores relatam a união destes nervos por meio de uma alça dupla cujo ramo mais caudal é muito delgado. Este nervo apresenta uma gama de descrições entre as espécies: na capivara ${ }^{23}$ sua origem varia entre C4-6 e C5-7, em pacas ${ }^{17}$ entre $\mathrm{C} 5$ e $\mathrm{C} 7$ e no porco-espinho ${ }^{19}$ se verificam as raízes cervicais $\mathrm{C} 5$ e C6. Entretanto, nos gatos ${ }^{5}$ e chinchila ${ }^{14}$, o nervo supra-escapular se origina somente do sexto nervo espinhal cervical (C6) e do sétimo nervo cervical em preguiça-de-coleira ${ }^{25}$.

No Cerdocyon thous o nervo subescapular é formado pelos ramos ventrais do sexto e sétimo nervos espinhais cervicais (C6 e C7), assim como ocorre no cão doméstico ${ }^{10}$, no gato ${ }^{5}$, no cateto ${ }^{13}$ e na chinchila $^{14}$. A origem deste nervo é diferente na 
capivara em que, segundo Fioretto et al. ${ }^{23}$, participam os segmentos C5 e C6 ou C5, C6 e C7, sendo esta última a mesma descrição encontrada para $\operatorname{ratos}^{26}$. Tal origem difere também do descrito para o cachorro-domato-de-orelhas-curtas ${ }^{11}$ e a paca ${ }^{17}$, em que o nervo subescapular é derivado somente do ramo ventral cervical de C6.

Segundo Ghoshal ${ }^{5}$, nos carnívoros, o nervo musculocutâneo tem uma origem variável. Ele normalmente deriva do ramo ventral do sétimo nervo cervical, mas pode receber uma pequena contribuição quer do sexto ou do oitavo nervos cervicais ou, em casos excepcionais, de ambos os nervos citados. No cão, a formação do nervo musculocutâneo procede das raízes ventrais cervicais $\mathrm{C} 6, \mathrm{C} 7$ e $\mathrm{C} 8{ }^{10}$, embora Sharp et al. ${ }^{27}$ considerem também a contribuição de T1.

Quanto ao Cerdocyon thous, tal nervo originou-se somente de C6 e C7, semelhante ao reportado para cachorro-do-mato-de-orelhas-curtas ${ }^{11}$ e chinchilas $^{14}$. Diferentemente do descrito para pacas ${ }^{17}$, em que a origem deste nervo também procede dos três últimos nervos espinhais cervicais, para catetos $^{13}$, no qual se origina de $\mathrm{C} 7$ e C8, e capivaras ${ }^{23}$ em que há participação de C6 a T1.

A origem do nervo axilar no Cerdocyon thous se da a partir de um ramo de $\mathrm{C} 7$ e $\mathrm{C} 8$ combinados, semelhante ao descrito para o cão doméstico ${ }^{10}$, e penetra no espaço entre o subescapular e o redondo maior ao nível do colo da escápula. Entretanto, ratos $^{26}$, gatos $^{5}$, chinchilas ${ }^{14}$, catetos $^{13}$, pacas ${ }^{17}$ e cachorro-domato-de-orelhas-curtas ${ }^{11}$ apresentam este nervo originado das raízes ventrais cervicais C6 e C7. Na capivara, a origem aparente do nervo axilar foi relatada a partir de $\mathrm{C} 5$ e $\mathrm{C} 6$ ou de $\mathrm{C} 6, \mathrm{C} 7$ e $\mathrm{C} 8$ e distribuído apenas ao músculo tríceps braquial ${ }^{23}$.

O nervo radial foi o único componente do plexo braquial do Cerdocyon thous que apresentou variação quanto à origem entre os animais estudados. Em um exemplar, tal nervo teve origem em $\mathrm{C} 7$ e C8 e nos demais, em C7, C8 e T1, apresentando três raízes, da mesma forma que no cão ${ }^{5}$, gato ${ }^{5}$, chinchila $^{14} \mathrm{e}$ paca $^{17}$. Nos cães, em alguns casos, pode haver também a contribuição de $\mathrm{T} 2^{10}$. Em catetos ${ }^{13}$, tal nervo é originado do oitavo nervo cervical e dos dois primeiros torácicos $(\mathrm{C} 8, \mathrm{~T} 1$ e $\mathrm{T} 2)$, enquanto que, na capivara, tem-se observado uma discrepância, já que nesta espécie o nervo tem origem de C6-8 e T1, ou seja, de todas as raízes que formam o plexo. No cachorro-do-mato-de-orelhas-curtas ${ }^{11}$ tal nervo tem origem em $\mathrm{C} 7$ e $\mathrm{C} 8$.

Os nervos mediano e ulnar do Cerdocyon thous são originados de um tronco comum formado pelo oitavo nervo cervical e primeiro torácico $(\mathrm{C} 8 \mathrm{e}$ $\mathrm{T} 1$ ), assim como na paca ${ }^{17}$. No cão ${ }^{10}$ também há a participação de T2 na formação desse tronco. No cateto $^{13}$ o nervo mediano é formado por $\mathrm{C} 7, \mathrm{C} 8, \mathrm{~T} 1 \mathrm{e}$ T2, e na chinchila ${ }^{13}$, por C7, C8 e T1. Já o nervo ulnar, no cateto $^{13}$, é formado por $\mathrm{C} 8, \mathrm{~T} 1$ e $\mathrm{T} 2$, enquanto que na chinchila ${ }^{14}$ o nervo é formado por C7 e T1. No cachorro-do-mato-de-orelhas-curtas ${ }^{11}$, o nervo radial teve origem em $\mathrm{C} 7$ e $\mathrm{C} 8$ e o nervo mediano em C7, C8 e T1.

O nervo toracodorsal do Cerdocyon thous tem sua formação estabelecida pelos nervos espinhais C8 e T1. Nos cães, surge do ramo ventral do oitavo nervo cervical, com contribuições do sétimo nervo cervical e do primeiro torácico ${ }^{5,10}$. Na capivara, na formação deste nervo há participação de $\mathrm{C} 6$ a T1 $1^{23}$ enquanto que na paca de $\mathrm{C} 8$ a $\mathrm{T} 2{ }^{17}$. No cachorro-do-mato-deorelhas-curtas $^{11}$, dos ramos ventrais C8 e T1 originaram os nervos ulnar, toracodorsal e peitorais caudais.

O nervo torácico lateral apresenta contribuição de C8 e T1 em sua formação, semelhantemente ao reportado para o cão $0^{5}$; entretanto, neste com contribuição inconstante de C7 e T2. Diferentemente, na capivara ${ }^{23}$ há contribuição de $\mathrm{C} 7$ a T2 e na paca ${ }^{17}$ de C8 a T2.

\section{CONCLUSÃO}

O plexo braquial do Cerdocyon thous originou-se a partir de comunicações entre os ramos ventrais dos nervos espinhais que emergem das três últimas vértebras cervicais e da primeira torácica (C6T1). Os nervos derivados do plexo braquial com suas respectivas origens foram: nervo supraescapular (C6C7), nervo subscapular (C6-C7), nervo musculocutâneo (C6- C7), nervo axilar (C7-C8), nervo radial (C7-T1 e C7-C8), nervo mediano (C8$\mathrm{T} 1)$, nervo ulnar (C8-T1), nervo toracodorsal (C8-T1) e nervo torácico lateral (C8-T1). Os animais objeto deste estudo assemelharam-se ao que foi descrito para animais domésticos no que se diz respeito aos seguimentos de origem do plexo braquial, apresentando diferenças quanto à composição de alguns nervos.

\section{REFERÊNCIAS}

1. Beisiegel BM, Lemos FG, Azevedo FC, Queirolo D, Jorge RSP. Avaliação do risco de extinção do Cachorro-domato Cerdocyon thous (Linnaeus, 1766) no Brasil. Biodiversidade Brasileira [Internet]. 2013; 3 (1):138-145. Available from: http://www.icmbio.gov.br/revistaeletronica/index.php/Bio BR/article/view/380/287. Portuguese.

2. Emmons LH. Neotropical rainforest mammals: a field guide. Chicago: The University of Chicago Press; 1999. 306p. English. 
3. Facure KG, Giaretta AA, Monteiro-Filho ELA. Food habits of the crab-eating fox, Cerdocyon thous, in an altitudinal forest of the Mantiqueira Range, Southeastern Brazil. Mammalia. 2003; 67:503-511.

4. Brady CA. Reproduction, growth and parental care in crab-eating foxes (Cerdocyon thous) at the National Zoological Park Washington. Internacional Zoo Yearbook [Internet]. 1978; 18:130-134. Available from: http://sipddr.si.edu/handle/10088/9795. English.

5. Ghoshal NG. Nervos espinhais. In: Getty R. Sisson/Grossman anatomia dos animais domésticos. 5nd ed. Rio de Janeiro: Guanabara-Koogan; 1986. p. $1597-$ 1600. Portuguese.

6. Dyce KM, Sack WO, Wensing CJG. Tratado de anatomia veterinária. 4nd ed. Rio de Janeiro: Elsevier; 2010. 856p. Portuguese.

7. König HE, Liebich HG. Sistema nervoso. In: Anatomia dos animais domésticos: texto e atlas colorido. Porto Alegre: Artmed; 2004. p. 203-275. Portuguese.

8. Carpenter MB. Neuroanatomia Humana. 7nd ed. Rio de Janeiro: Interamericana; 1978. 770p. Portuguese.

9. International Committee on Veterinary Gross Anatomical Nomenclature. Nomina anatômica veterinária. 5nd ed. Knoxville: World Association on Veterinary Anatomist; .[Internet] 2012. 160p. Available from http://www.wava-amav.org/committees_icvgan.htm English.

10. Evans HE, de Lahunta A. Guia para dissecção do cão. 5nd ed. Rio de Janeiro: Guanabara Koogan; 2001. p. 103 109. Portuguese.

11. Pinheiro LL, Souza DC, Branco É, Souza ACB, Pereira LC, Lima AR. Descrição do plexo braquial do cachorro-domato-de-orelhas-curtas (Atelocynus microtis - Sclater, 1882): relato de caso. Biotemas [Internet]. 2013; 26(3):203-209. Available from: http://dx.doi.org/10.5007/2175-7925.2013v26n3p203.

Portuguese.

12. Allan MW, Lee DG, Nulsen FE, Fortune EA. The anatomy of the brachial plexus of the dog. The Anatomical Record [Internet]. 1952;114:173-180. Available from: http://onlinelibrary.wiley.com/doi/10.1002/ar.1091140205 /pdf. Portuguese.

13. Moura CEB, Albuquerque JFG, Magalhães MS, Silva NB, Oliveira MF, Papa PC. Análise comparativa da origem do plexo braquial de catetos (Tayassu tajacu). Pesquisa Veterinária Brasileira [Internet]. 2007;27(9):357-362. Available from: http://dx.doi.org/10.1590/S0100736X2007000900001. Portuguese.

14. Gamba CO, Castro TF, Rickes EM, Pereira MAM. Sistematização dos territórios nervosos do plexo braquial em chinchila (Chinchilla lanigera). Brazilian Journal of Veterinary Research and Animal Science [Internet]. 2007; 44(4):283-289. Available from: http://www.revistas.usp.br/bjvras/article/view/26629/2841 2. Portuguese.
15. Santana JJ, Albuquerque JFG, Moura CEB, Costa WP, Oliveira MF, Barreto Júnior RA, Miglino MA. Origem do plexo braquial de mocós (Kerodon rupestris Wied, 1820). Brazilian Journal of Veterinary Research and Animal Science [Internet]. 2003;40:391-396. Available from: http://dx.doi.org/10.1590/S1413-95962003000600001.

Portuguese.

16. Cruz GAM, Adami M, Almeida AEFS, Silva EAAC, Faria MD, Pinto MGF, et al. Características anatômicas do plexo braquial de tamanduá-mirim (Tamandua tetradactyla Linnaeus, 1758). Revista Brasileira Saúde Produção Animal [Internet]. 2012;13(3):712-719. Available from: http://revistas.ufba.br/index.php/rbspa/article/viewArticle/ 2474. Portuguese.

17. Scavone ARF, Machado MRF, Guimarães GC, Oliveira FS, Gerbasi SHB. Análise da origem e distribuição dos nervos periféricos do plexo braquial da paca (Agouti paca Linnaeus, 1766). Ciência Animal Brasileira [Internet]. 2008;9(4):1046-1055. Available from: http://www.revistas.ufg.br/index.php/vet/article/view/110 $\underline{0 / 4366}$. Portuguese.

18. Ribeiro AR, Prada ILS, Silva Z, Barros RAC, Silva DCO. Origem do plexo braquial do macaco Cebus apella. Brazilian Journal of Veterinary Research and Animal Science [Internet]. 2005; 42(2):143-149. Available from: http://www.revistas.usp.br/bjvras/article/view/26445. Portuguese.

19. Aydin A. Brachial plexus of the porcupine (Hystrix cristata). Veterinari Medicina [Internet]. 2003; 48(10):301304. Available from: http://agris.fao.org/aos/records/CZ2004000493. English.

20. Uzun A, Cengiz N, Kavakli A, Karakas S. Morphological and microscopical examination of the rat brachial plexus. Turk-Veterinerlik-ve Hayvancilik-Dergisi. 2001; 25:397-402.

21. Koizumi M, Sakai T. On the morphology of the brachial plexus of the platypus (Ornithorhynchus anatinus) and the echidna (Tachyglossus aculeatus). Journal of Anatomy [Internet]. 1996; 190:447-455. Available from: http://www.ncbi.nlm.nih.gov/pubmed/9147230. English.

22. Koizumi M, Sakai T. The nerve supply to coracobrachialis in apes. Journal of Anatomy [Internet]. 1995; 186:395-403. Available from: http://www.ncbi.nlm.nih.gov/pmc/articles/PMC1167198/p df/janat00133-0156.pdf. English.

23. Fioretto ET, Castro MFS, Guidi WL, Mainardi R, de Souza RR, Ribeiro AACM. Gross anatomic organization of the capybara's brachial plexus (Hydrochaeris hydrochaeris). Anatomia Histologia Embryologia [Internet]. 2003; 32:169-174. Available from: http://www.ncbi.nlm.nih.gov/pubmed/12823103. English.

24. Bielik P. Constituição do Plexus Brachialis no Bradypus tridactylus. Arquivos de Anatomia e Antropologia. 1937; 18:1-4.

25. Cruz GAM, Adami M, Oliveira VL. Características anatômicas do plexo braquial de bicho-preguiça-de-coleira 
(Bradypus torquatus Illiger, 1811). Biotemas [Internet]. 2013; 26(3):195-201. Available from: http://dx.doi.org/10.5007/2175-7925.2013v26n3p195. Portuguese.

26. Greene EC. Anatomy of the rat. New York: Hafner Publishing Company; 1955. p. 127-169. English.

27. Sharp JW, Bailey CS, Johnson RD, Kitchell RL. Spinal nerve root origin of the median, ulnar and musculocutaneos nerves and their muscle nerve branches to the canine forelimb. Anatomia Histologia Embryologia [Internet]. 1990;19:359-368. Available from: http://www.ncbi.nlm.nih.gov/pubmed/2077954. English.

Protocolado em: 25 set. 2012 Aceito em: 22 abr. 2014. 